\title{
Centralized Relay Selection and Optical Filtering Based System Design for Reliable Free Space Optical Communication over Atmospheric Turbulence
}

\author{
M. Mubasher Hassan \\ Research Scholar, ECE Department, National Institute of Technology (NIT), \\ Srinagar, J\&K - 190006 \\ Assistant Professor BGSB University Rajouri (J\&K) \\ E-mail: mubasher2003@gmail.com \\ G M Rather \\ Professor, ECE Department, National Institute of Technology (NIT), \\ Srinagar, J\&K, India - 190006 \\ Email: gulammohdrather@yahoo.co.in
}

Received: 03 October 2019; Accepted: 20 December 2019; Published: 08 February 2020

\begin{abstract}
The need for much better data rate in support for the applications having the high speed has gathered the attention to the development of Free space Optical communication technology (FSO). But the widespread usage of this technology is restrained by the several ill effects caused by the atmosphere on the propagation of optical signals. In order to mitigate such problems in the FSO communication, relay assistance has been employed and this serves the alternative to the spatial diversity. Free space optical (FSO) communication is a cost-effective and high bandwidth access technique, which has been receiving attention to recent commercialization successes. FSO get affected by the weather turbulence and it leads to severe problems in the transmission channels. In this paper, we proposed the Centralized Relay selection and Optical Filtering (CROF) for processing the signals in the FSO system which overcomes the environmental challenges. Initially, an effective relay is selected with the centralized switch and stay algorithm. This algorithm selects suitable relay for serial path transmission. Here, the serial path is considered for signal transmission in a free path and the distributed Greedy algorithm with serial transmission is utilized to obtain more information. Then atmospheric turbulence induced scintillation noise presented at the signal is eliminated through modified Kalman filtering. Finally, the performance of the proposed FSO system is evaluated in MATLAB simulation platform and these evaluations are analyzed for different atmospheric conditions like fog, haze, rain and clear weather. The performance evaluation shows that the proposed algorithm outperforms the noncooperative scheme and an existing relay selection protocol and reliable communication with improved SNR and BER is achieved.
\end{abstract}

Index Terms-Free Space Optical Communication, Atmospheric turbulence, relay selection, weather conditions, serial model, filtering.

\section{INTRODUCTION}

Free space optical communication (FSO) has gained significant attention in research and in commercial applications due to its unique features like license free spectrum, large bandwidth and low cost $[1,2]$. The FSO mode of wireless technology was not implemented at large till date due to its poor performance under adverse atmospheric conditions and the corresponding need was fulfilled by RF technology. But now the demand for large bandwidth for future communication systems cannot be fully satisfied by RF and therefore, the paradigm shift towards FSO communication comes into the picture. The major challenge in FSO communication is to mitigate the ill effects of various atmospheric conditions. The FSO system performance depends intensely on characteristics of the channel and the atmosphere in turn decides the channel characteristics. Thus, for a point-to-point optical link, the inhomogeneities in temperature causes variations in refractive index leading to atmospheric turbulence. This degrades the communication system performance mainly over the distances of $1 \mathrm{~km}$ or longer [3].

In order to describe the characteristics of optical channel, numerous statistical models have been proposed with reference to strength of atmospheric turbulence [2]. For modeling the irradiance of the optical channels for weak-to-moderate and moderate-to-strong turbulence channels, the log-normal as well as the gamma-gamma distributions have been found to be suitable; meanwhile 
they provide good agreement between theoretical and experimental data [4].

FSO communication is an important area in free-space information systems [5]. This technology is utilized for ship to ship communication, up-and-down connections among space platforms, aircraft, satellite-to-satellite cross links, other ground platforms, and between mobile terminals inside the environment [6]. In the free space environment, the various techniques have been adopted for turbulence management over FSO systems. If the medium includes broadcast over the environment and numerous applicants are distributed in the identical optical channel, the medium reveals arbitrary activities that relate compactly through protocols in the upper layer [7]. A different system designs are required to be enhanced through numerous system layers towards moderate system performance [8].

FSO is considered as the highly secure transmission medium and the main challenge of this technology is posed by the atmospheric weather condition [9]. Here signal scattering occurs due to the optical photon and this is caused by the small particles present in the air. If the particle size is comparable to the wavelength of optical wave, then scattering will occur. Since the particles are having larger size and it is more effective to the shorter wavelength of light [10]. In case of atmospheric attenuations like haze and fog, the visibility $(\mathrm{km})$ is considered as a significant weather parameter and it causes power loss [11]. If snow and rainfall are combined, then the dominant attenuation will occur. Additional experiments were performed in which the attenuation is described as a consequence of scattering [12]. FSO link unavailability is mainly caused due to heavy rain and dense fog in the tropical regions [13].

The most influences of a data link in FSO is due to variations of turbulence such as arbitrary optical power variation, optical absorption and scattering, displacement of the beam centroid and wavefront alterations. Additional influences happening due to environment (i.e. Weather data) are used for further processing [14]. In traditional processing, some methods are used for eliminating the challenge in FSO broadcast. The methods are Kim Model, Kruse Model, Al-Naboulsi Model and Ijaz Model [15]. The Kruse model has been widely used in recent years as the unique model that predicts the attenuation from the visibility data. One of the unique models for predicting attenuation problem is Kruse model that provides the visibility of data. Fog attenuation for Kim model is $\mathrm{V}<500 \mathrm{~m}$ which is considered as wavelength independent [16]. Attenuation of fog has the spectral band of $0.69-1.55 \mu \mathrm{m}$ and it has the visibility in the range between $50 \mathrm{~m}$ and $1 \mathrm{~km}$. Attenuation wavelength $\mathrm{V}>15 \mathrm{~m}$ for Ijaz Model and compared to infrared wavelengths, visible wavelengths are more attenuated [17].

The major challenge in the research is environmental condition which produces problems in the overall performances. The clear air turbulence also induces some problems and efficient coherent systems are needed to eliminate these problems. They also reduce the cost of communication and other interference problems [19]. Subsequently, these sources of FSO network improve the effective capacity of overall communication systems [20]. Problem regarding the existing works:

Transmission of optical signal is subjective through many challenges before it reaches to the receiver such as background noise, geometric losses, atmospheric turbulence, misalignment errors and weather attenuation losses. FSO communications are decided in dual trial facing process: which are weather attenuation and atmospheric turbulence by the geometric loss. This kind of loss is termed to power loss. Some literatures from [21-25] have the solution to solve the problem but still it has disadvantages.

The problem related to the existing works are worthy to note that in dense fog, using high power and/or shorter links could not help much to improve the system performance. So, high power consuming and low error interfering systems must be designed in proposed work to overcome the FSO challenges [35]. In this work we can solve the problem regarding data rate, throughput and SNR with RMSE values under haze, fog, rainy and clear atmosphere condition. For that we design CROF technique to achieve better model for solving the FSO difficulties.

Optical signal transmission is influenced by several difficulties before it reaches to the receiver such as geometric losses, background noise, atmospheric turbulence, misalignment errors and weather attenuation losses. FSO transmissions are conceived with two major attenuation problems and they are atmospheric turbulence and weather attenuation. Both these attenuation problems eventually lead to power loss. The work is based on FSO communication using relay assistance. Numbers of relays are distributed between the source and destination and the proposed relay selection algorithm outperforms some existing relay selection techniques.

The contribution of the work is given below:

The prior works on the optimal relay selection or relay placement in FSO networks were mainly focused on maximizing the diversity gain, reducing the outage probability, or maximizing the capacity for an individual BS. In this paper, we consider the challenging problem of relay selection and power allocation under power and cost constraints, aiming to maximize the overall FSO network capacity. We develop an effective method for minimizing scattering losses and this will lead to power of minimum atmospheric turbulence. This is highly competitive solutions to maximize the total throughput of the cooperative FSO network. The modified Kalman filter which progresses the performance in atmospheric turbulence based scintillation noise removal is the chief contribution of this paper. The choice of modulation schemes depends on two main criteria in the FSO communication, namely optical power efficiency and bandwidth efficiency. By computing optical power gain over OOK provided both, the modulation schemes have same Euclidean distance, the optical power efficiency can be measured. So compared to other techniques we have been using modified Kalman filter based scintillation 
noise removal techniques since we can achieve minimum Bit Error Rate (BER) which is given at section 5. In addition to that, we can also achieve better data rate, throughput and SNR with minimum RMSE values under haze, fog, rain and clear atmosphere condition. The experimental expectations are to establish a reliable link between source and destination under various environmental conditions for short range that can be used for various applications in the next generation networks. In our method, the transmitter transmits data from the source directed towards the relays. Subsequently, one relay forwards the signal and then retransmits it to the final destination. The best relay should have the highest end-to-end signal-to-noise ratio (SNR). In the SourceRelay scheme, there is a number of FSO centralized parallel relays in the communication system. The best relay selection is determined when the data is transmitted from source to the best relay. However, the concept of best relay selection can also be applied to coherent modulation and demodulation. This paper concentrates on IM/DD due to its simplicity and low cost. A different number of relays are considered. Moreover, different turbulence schemes are considered as the weak-moderate turbulence, which is modeled by $\mathrm{K}$ distribution channel model. The obtained results show that there is no optimum technique under different conditions, since the optimum technique depends on several factors, such as the number of relays, turbulence, and pointing bit error effects.

The objective of the work is given below:

- To design an efficient free space optical communication systems in the presence of weather attenuation losses and atmospheric turbulence

- To minimize the effects of scattering in FSO.

- To design the technique for the efficient relay selection

The remainder of this manuscript is organized as follows. In Section 2, we review the works related to the FSO communication over environmental factors. Section 3 describes the system model of the proposed work. In Section 4, the proposed methodology for improving reliable optical communication is described. The experimental setup of our evaluation is presented, strategies for combining relative criteria are discussed and the obtained results are discussed in Section 5. Section 6 addresses the conclusion of the paper.

\section{RELATED WORKS}

1. Imran Shafique Ansari et al. [21] framed the analysis of FSO system which resolves the issue of error reduction and it provides intensity modulation/direct detection (IM/DD). Additionally, they presented integrated expressions of the moment generating function, probability density function, cumulative distribution function and the moments of the SNR for FSO communication systems. Here asymptotic expansion of the Meijer's $G$ function also used for solving SNR problems.

2. Ambreen Niaz et al. [22] introduced a chaos masking scheme to hide the message signal inside the generated chaos before transmission. As FSO communication efficiency and link availability in different atmospheric conditions was a major concern. The FSO communication was investigated under fog, rain and haze conditions and analysis and comparison was made based on Q-factor and eye diagram pattern. The outcomes were further studied for both FSO communication models, i.e, with and without applying chaos masking scheme.

3. Abhijeet Upadhya et al. [23] developed a novel as well as an accurate mathematical expression of the probability density function for an FSO link experiencing $\alpha-\mu$ distributed atmospheric turbulence in the presence of pointing error. Further, an analytical expression of outage probability and bit error rate in terms of Meijer-G function was also presented. The atmospheric turbulence, various binary modulation schemes, misalignment errors for intensity modulation on the optical wireless link were considered to yield the results.

4. Ahmed Basahel et al. [24] suggested a technique which is suitable for FSO link availability in case of tropical climate. In this work they considered three cases for effective availability of the link (i.e. Dense haze, normal haze, and rain with dense haze). In case of dense, the attenuation is almost $155 \mathrm{~dB} / \mathrm{km}$ and for normal case it is $6 \mathrm{~dB} / \mathrm{km}$.

5. Maged Abdullah Esmail, et al. [25] introduced a probabilistic system under stochastic fog conditions. Here channel behaviours in various locations are measured in which the cell sizes are less than $1 \mathrm{~km}$. This system was suitable for fifthgeneration/sixth-generation $(5 \mathrm{G} / 6 \mathrm{G})$ networks because of less cell size.

6. The problem related to the existing works is worthy to note that in dense fog, using high power and/or shorter links could not help much to improve the system performance. So, high power consuming and low error interfering systems must be designed to overcome the FSO challenges.

The literature survey gives an impression for developing various FSO systems using different approaches. The common point in these works relies on an improvement in performance, so as to establish reliable optical wireless connectivity. Different performance parameters like SNR, Q-factor and BER were analyzed in different weather conditions. The objective of the proposed work is related to the literature taken into consideration with the intention to improve further performance. 


\section{SYSTEM MOdel FOR THE PROPOSED FSO}

Centralized Relay selection and Optical Filtering (CROF) for modelling an FSO system is designed in our work to overcome environmental challenges. The modified Kalman filter, analyse the noise performance of both single-stage and dual-stage filters by evaluating the signal to noise ratio. A perfectly fast-tracked FSO laser communication system experiences random power fading due to atmospheric turbulence. For an FSO communication system without fast-tracking or with imperfect fast-tracking, the fading probability density function is affected by the pointing error. The pointing error is the overall displacement between the laser beam centre and the receiver aperture centre.

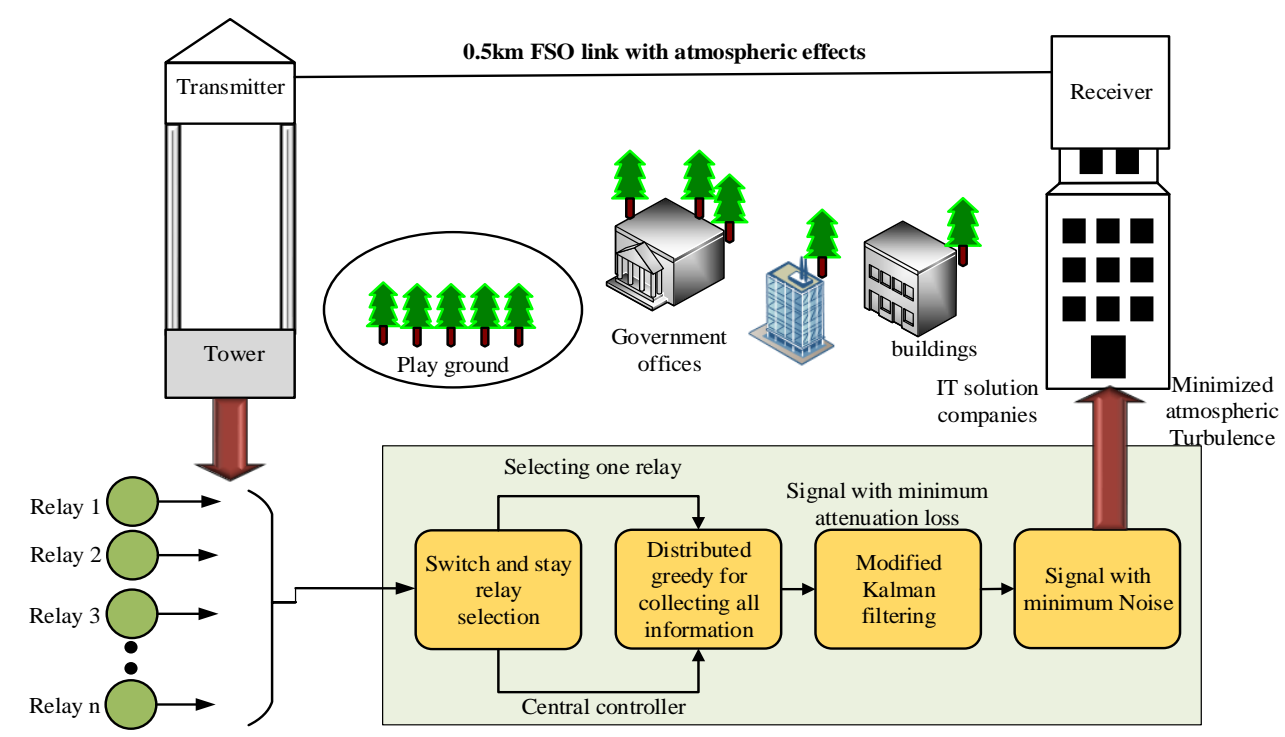

Fig.1. Schematic representation of proposed CROF system

Schematic representation of the proposed CROF system is represented in figure 1. In this paper, we consider the challenging problem of relay selection in FSO networks. The objective is to maximize the throughput of FSO network under constraints of a given power budget and a limited number of FSO transceivers. Here relay selection avoids the power loss and it is accomplished through a distributed switch and stay relay selection algorithm. The centralized relay selection algorithm requires a central controller. For that, we used the distributed greedy selection algorithm. Finally, scintillation noise due to atmospheric turbulence is eliminated with the modified Kalman filtering.

The FSO communication system includes single transmitter, receiver in addition to multiple relays. Among them best relay is selected which is extensively structured in FSO systems. Here atmospheric turbulence mitigation is processed with On-Off Keying (OOK) modulation and Modified Kalman filtering techniques.

\section{A. System model}

Reliable Free space optical (FSO) communication is investigated as efficient FSO transmission. Atmospheric turbulence effects cause fluctuation in the optical power. A suitable power link margin is required to overcome the power fluctuations. This evaluation is carried out in the presence of two difficulties such as atmospheric turbulence and weather attenuation losses under various atmospheric conditions. In addition to the atmospheric turbulence, optical signal scattering is also required to be reduced with the suitable mitigation technique. In FSO, base stations use relays structures to improve the link quality. The system model under consideration is depicted in Fig. 1. In particular, the communication between the source $\mathrm{S}$, and the destination $\mathrm{D}$, is achieved with the aid of multiple relays, denoted by $R_{i}, i \in\{1, \ldots N\}$.

\section{B. Channel model}

$\mathrm{K}$-distribution channel model is utilized here, as it is suitable for strong turbulent regimes [36]. This offers experimentally and practically good results in the system. The K-channel could not easily relate the channel parameters with atmospheric turbulences. Therefore, it has limited utilization as well as applications. The $\mathrm{K}$ distribution channel model is appropriate to achieve a distance of $1 \mathrm{~km}$.

High directional FSO links are identically susceptible to weather turbulence and it causes signal degradation due to propagation. In this proposed method, FSO link turbulence and power loss are taken into account for modelling an entire framework. For that, initially an optical channel state $\mathrm{S}$ is designed and it is a combination of two factors (i.e., atmospheric turbulence impacts $\left(\mathrm{S}_{1}\right)$ and propagation loss $\left(\mathrm{S}_{\mathrm{f}}\right)$ ) as [26].

$$
s=s_{l} \times s_{f}
$$

In this above equation (1) turbulence is the function of link length (l) and optical wavelength $(\lambda)$. Then the $S_{1}$ function is given in below equation (2). 


$$
s_{l}=\frac{A_{T x} B_{R x} e^{-\alpha l}}{(\lambda . l)^{2}}
$$

In equation (2), $A_{T x}$ and $A_{R x}$ represent the aperture areas of transmitter and receiver respectively. The attenuation coefficient $(\alpha)$ is the relational component of the environment and wavelength. In case of fading effects, $\mathrm{S}_{\mathrm{f}}$ is modelled and it can be mostly used for weak and moderate turbulence.

The FSO channel model is written as in (3)

$$
y=s \cdot x+n
$$

In this above equation, transmitted and received signals are represented as $\mathrm{x}$ and $\mathrm{y}$. The noise is denoted as ' $\mathrm{n}$ '. Here, the maximum data rate is obtained with the communication pair and this is given below in equation (4).

$$
w_{h, l}=B \log \left(1+\frac{\left|s_{h, l}\right|^{2} W_{h}}{\sigma^{2}}\right)
$$

The source and destination direct link are given as $S_{h, l}$ and transmit power is given as $\mathrm{W}_{\mathrm{h}, \mathrm{l}}$. Noise power is induced from the channel during the transmission which is given as $\sigma^{2}$. Thus, the maximum achievable capacity between both sender and receiver is demonstrated as $\mathrm{P}_{\mathrm{s}, \mathrm{r}}$.

$$
P_{s, r}=\frac{B}{2} \min \left\{\log _{2}\left(1+\frac{\left|s_{h, r}\right|^{2} W_{h}}{\sigma^{2}}\right), \log _{2}\left(1+\left(\frac{\left|s_{h, l}\right|^{2} W_{h}}{\sigma^{2}}+\frac{\left|s_{r, l}\right| W_{r}}{\sigma^{2}}\right)\right)\right\}
$$

In equation (5), $\mathrm{W}_{\mathrm{h}}$ and $\mathrm{W}_{\mathrm{r}}$ denotes the transmit power of relay's sender and receivers. B denotes the bandwidth and $S_{\mathrm{h}, \mathrm{r}}$ and $S_{\mathrm{r}, \mathrm{l}}$ are the channel states of source and destination relay link. A different channel model comparison is given in table 1 [38].

Table 1. Comparison of different channel models

\begin{tabular}{|c|c|c|c|}
\hline Model & Parameters & Atmospheric turbulence & Findings \\
\hline $\begin{array}{c}\text { Kolmogrov } \\
\text { spectrum model }\end{array}$ & $\begin{array}{c}\text { 1550nm wavelength } \\
\text { Infinite radius of curvature } \\
\text { OdBm transmitted power }\end{array}$ & $\begin{array}{c}\text { Weak } \\
\left(\sigma^{2} \leq 0.3\right)\end{array}$ & $\begin{array}{c}\text { For } 2 \mathrm{~km} \text { distance this model is } \\
\text { suitable }\end{array}$ \\
\hline $\begin{array}{c}\text { Log normal model } \\
\text { Receiver diameter } 0.1 \mathrm{~m} \\
\text { Temperature } 300 \mathrm{~K}\end{array}$ & $\begin{array}{c}\text { Weak } \\
\left(\sigma^{2} \leq 0.3\right)\end{array}$ & $\begin{array}{c}\text { Average transmitted power is } \\
\text { improved }\end{array}$ \\
\hline $\begin{array}{c}\text { Attenuation coefficient } 0.1 \mathrm{~dB} / \mathrm{Km} \\
\text { Log normal channel }\end{array}$ & $\begin{array}{c}\text { 1550nm wavelength } \\
\text { Transmitted power } 26 \mathrm{dBm} \\
\text { Bandwidth } 0.5 \mathrm{GHz}\end{array}$ & $\begin{array}{c}\text { Weak } \\
\left(\sigma^{2}=0.297\right)\end{array}$ & $\begin{array}{c}\text { Link value is 3.45 Km under weak } \\
\text { turbulence condition } \\
\text { Availability is above } 99.9 \% .\end{array}$ \\
\hline $\begin{array}{c}\text { Gamma Gamma } \\
\text { channel distribution }\end{array}$ & $\begin{array}{c}\text { System temperature is } 300 \mathrm{~K} \text { in } \\
\text { atmospheric turbulence. }\end{array}$ & $\begin{array}{c}\text { Moderate to strong } \\
\left(0.25<\sigma^{2}<0.75\right)\end{array}$ & $\begin{array}{c}\text { Spectral efficiency rate is decreased } \\
\text { with increasing transmitted power. }\end{array}$ \\
\hline $\begin{array}{c}\text { K distribution } \\
\text { channel model }\end{array}$ & $\begin{array}{c}\text { Wavelengths } 1550,1310,850 \mathrm{~nm} \\
\text { Link distance } 2.5 \mathrm{~km}, 3 \mathrm{~km} .\end{array}$ & $\begin{array}{c}\text { If the number of wavelengths } \\
\text { increases with outage probability } \\
\text { decreases. }\end{array}$ \\
\hline
\end{tabular}

\section{Turbulence model}

In an optical link, due to the change of refractive index of an air may produce significant fluctuation in the signal propagation. Thus the coherence of the receiver is reduced and it produces some fluctuation during the processing of optical signal. Usually some distributions are modelled to characterize the turbulence effects (i.e strong, moderate and weak). Following expression gives the probability density function of turbulence [26].

$$
P_{G}(x)=\frac{2(\sigma \rho)^{(\sigma+\rho) / 2}}{\Gamma(\sigma) \Gamma \rho} \alpha^{(\sigma+\rho / 2)^{-1}} B_{\sigma+\rho}(\sqrt[2]{\sigma \rho \alpha})
$$

Where $\alpha$ is the attenuation coefficient of transmitted signals and the interference and it should be greater than zero. Effective large and small scale eddies are denoted by $\sigma$ and $\rho$ and $\Gamma$ represents the gamma function of the distribution and $\mathrm{B}$ is the modified Bessel function. In each case, gamma function should be treated independently to eliminate the effect of turbulence. The turbulence metric is represented as the difference among received mean irradiance to the standard deviation of the irradiance, and enlarging metric stabilities the belongings of scintillation and beam spread [29]. A Gaussian Schellmodel beam is established through the arrangement of metric, in which the optimization parameter is the transverse coherence length. The metric is realistic for establishing the features of the enhanced coherence length for a purpose of a variety of link parameters and scenarios. In general, the optimized coherence length tends to decrease with maximizing propagation distance and turbulence strength. Thus, $P_{G}(x)$ parameters aimed at best performance in an FSO link should balance the development for reducing scintillation through the loss of signal strength due to divergence.

\section{Centralized Relay SElection AND Optical FILTERING IN FSO}

DSSRS algorithm is mainly used for the relay selection process and it is one of the centralized algorithms for the power loss control. Here synchronization among the relays is not possible since it uses end to end time slot 
activation at a time and here end to end path is only active at a time of transmission. Significance of utilizing relay selection in multiple relayed scheme in the multiple relayed system is that there is no need of synchronisation among relays, as only one end-to-end path is active in each transmission slot.

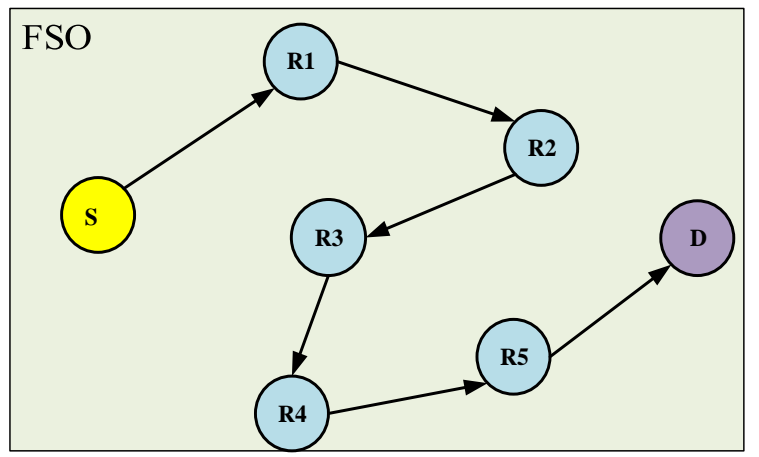

Fig.2. FSO system with optimal relay

In the above fig.2., serial relay path is given and this will be selected through a hybrid switch and stay and greedy selection algorithm.

The deployment of relays is such that there is the possibility of multiple paths through one or more than one relay between source and destination. Fig. 2. Showed that all the $\mathrm{R}$ relays has Loss of signal propagation regarding forwarding capacity within one way communication. Since, the channel impairments are depend on distance. All terminal node is termed with antenna focused to the $\mathrm{R}$ relays, in order to carry out exchange of data among transmitter and receiver, best relay is selected out of the $\mathrm{R}$ relays using the proposed relay selection algorithm. The following technical highlights are being incorporated;

> The path is selected based on an end to end SNR.

$>$ The only single path is selected at one time for transmission of data.

> The SNR of the path chosen is continuously sensed and if it falls below the threshold, another path is immediately switched over.

> This topology can also be used in case source and destination are in non line of sight (NLOS), but transmission from one node to another node must be in LOS.

In serial relaying (Fig 2), the source sends an intensity modulated signal to a relay node, which further forwards to another relay. After multiplication through an efficient energy scaling term, just send it to the next relay. This remains till the source's information reaches at the destination node.

\section{A. Switch and Stay algorithm for Relay Selection}

This algorithm is suitable for more than two relays and these relays are sorted by the distance estimation criteria. Let us consider the number of relays which is greater than two (i.e $\mathrm{N}>2$ ). After setting the number of relays, the main components are sorted with some distance

$$
d_{n}=\max \left(d_{h r_{n}}, d_{d r_{n}}\right) \text { Where } n=1,2, \ldots, N
$$

The source distance measure is denoted as $d_{h r_{n}}$ and the destination distance is denoted as $d_{d r_{n}}$ Next to the sorting process, the minimum distance is estimated with Manhattan distance and which is given in the above equation (7). From this distance estimation criteria relay with maximum distance is selected for next processing. The major objective of this algorithm is to select a unique relay among a number of relays then only communication is possible between two nodes. In this method one switching threshold $\left(\tau_{s w}\right)$ is considered for the active transmission and in addition to this, each time destination compares the equivalent end to end $\operatorname{SNR}\left(\tau_{e q}\right)$. Relay selection is given as in the below equation (8).

$$
\text { If } R_{n-1}^{s e l}=R_{1} \text {, then } R_{n}^{s e l}=\left\{\begin{array}{l}
R_{1}, \text { for } \tau_{e q} \geq \tau_{s w} \\
R_{2}, \text { for } \tau_{e q}<\tau_{s w}
\end{array}\right.
$$

Where, $R_{n-1}^{\text {sel }}$ denotes the selected relay at $n-1^{\text {th }}$ slot of transmission, $R_{n}^{\text {sel }}$ represents the selected relay at $n^{\text {th }}$ slot of transmission. $R_{1}$ and $R_{2}$ denotes the relay. If equation (8) is possible for nth relay and if it is not suitable then we have to consider the condition in equation (9).

$$
\text { If } R_{n-1}^{s e l}=R_{2}, \text { then } R_{n}^{s e l}=\left\{\begin{array}{l}
R_{2}, \text { for } \tau_{e q} \geq \tau_{s w} \\
R_{1}, \text { for } \tau_{e q}<\tau_{s w}
\end{array}\right.
$$

Based on the above mentioned conditions, relay is selected which is given in equation (9) and it should be presented at the nth time slot. For the active relay selection one condition is estimated and according to this condition, best relay should have the value (i.e, SNR should be greater than the switching threshold). This switch and stay protocol is more simple based on the fact that only the Channel State Information (CSI) of the active end-to-end path is required, resulting in lower complexity in implementation.

Next to relay selection central controller is used for further processing. Thus single relay is selected among multiple relays in the given time slot. Based upon the optimum relay selection, diversity gain is maximized and outage probability is reduced.

\section{B. Distributed Greedy for information gathering}

Greedy algorithm is mainly used for gathering the maximum information about the channel and the base station of each transmission uses central state information. Here, the problem of power allocation is solved in a distributed manner and some of local variables are introduced for the power allocation in FSO communication. Here the power range of $p_{i, i}$ and $p_{i, j}$ are introduced for the efficient signal propagation. In 
addition to the local variables, here we introduce some auxiliary variables for localizing the capacity of relay's power allocation. Thus the power allocation problems are given as in the following expression (10).

$$
\begin{gathered}
P=\max \sum_{i \in S_{1}} s_{h, l}^{i, i}+\sum_{i \in S_{2}} t_{1}^{*} \\
P=\sum_{j=1}^{N} p_{i, j} \leq \bar{p}
\end{gathered}
$$

Where, $s_{h, l}^{i, j}$ is a logarithmic function, $t_{1}^{*}$ denotes the auxiliary variables. From the above equation (11), the value of power in the distributed network should be within minimum power. In case of variable power, it should have the limit within the maximum power for the entire transmission.

$$
P_{s, r}=\frac{B}{2} \min \left\{\log _{2}\left\{1+\frac{\left|s_{i, r_{i}}\right|^{2} p_{i, i}}{\sigma^{2}}\right\} \geq t_{i}^{*}, \forall i \in S_{2}\right\}
$$

in which $S_{i, r_{i}}$ denotes the channel state of the direct link between source and destination, $P_{i, i}$ is the transmit power, and $\sigma^{2}$ is the noise power. When one relay is used, which can be expressed as

$$
P_{s, r}=\frac{B}{2} \min \left\{\log _{2}\left(1+\frac{\left|s_{i, i}\right|^{2} p_{i, i}}{\sigma^{2}}+\frac{\left|s_{r_{i}, i}\right|^{2} p_{i}^{*}}{\sigma^{2}}\right) \geq t_{i}^{*}, \forall i \in S_{2}\right\}
$$

Where $P_{i, i}$ and $P_{i}^{*}$ denoted the source and relay's transmit power, and $s_{i, i}$ and $s_{r_{i}, i}$ are the channel positions of the sender relay link and the relay-target link, respectively.

$$
\begin{aligned}
& t_{r_{i}, i}=t_{i}^{*}, t_{j, i}=0, \forall i \in S_{2} \\
& P_{r_{i}}, i=P_{i}^{*}, p_{i, j}=0, \forall i \in S_{2}
\end{aligned}
$$

The equation (14) represents that BS transmits directly to its destination BS without using any relay. $P_{r_{i}, i}$ is the power allocation process which is functioned by the relay. Then $S_{1}$ and $S_{2}$ are set which accomplishes the transmission with or without relay. Then, the modified power allocation algorithm is used for the power distribution throughout the entire communication. Karush-Kuhn-Tucker (KKT) conditions are considered as the decomposition solutions for the local variables. So the set with relay source have local maximization problem which is expressed in equation (16).

$$
\max K_{1 i}=w_{h, d}^{i, i}+\lambda_{1, i}\left(\bar{P}-\sum_{j} P_{i, j}\right)+\mu_{1, i}^{T} t_{i}+\mu_{2, i}^{T} P_{i}, \forall i \in \ell_{1}
$$

Where, $\mu_{i}$ and $\lambda_{i}$ are the two KKT transforms and the power allocation vector is given as $\bar{p}_{i}$, the value of allocation vector is the transpose of an allocation matrix. $\bar{p}_{i}=\left[p_{i, 1}, p_{i, 2}, \ldots p_{i, n}\right]^{T}$. Thus the maximization problem is achieved through equation (16) and in case of relay source set relay selection is the complex problem and thus power allocation is determined at the base station.

For the relay source, the maximization problem is given by the below equation (17)

$$
\begin{aligned}
\max K_{2 i} & =t_{i}^{*}+\sum_{i=1}^{n} \mu_{i}\left(\overline{p_{i}}-\sum_{j} p_{i, i}\right)+\sum_{j=1}^{m} \lambda_{1, i}\left(-\bar{p}_{i}-\sum_{j} p_{i, j}\right)+\lambda_{2, i}\left(\frac{B}{2} \log _{2}\left(1+\frac{\left|s_{i, r_{i}}\right|^{2} p_{i, i}}{\sigma^{2}}\right)-t_{i}^{*}\right) \\
& -\mu_{1, r_{i}, i} \times t_{i}^{*}-\mu_{2, r_{i}} \times p_{i}^{*}+\mu_{1, i}{ }^{T} \times t_{i}^{*}-\mu_{2, i}{ }^{T} \times p_{i}^{*}
\end{aligned}
$$

Where $\lambda_{1, i}, \lambda_{2, i}, \mu_{1, i}, \mu_{2, i}, \mu_{i}$, are the KKT multipliers and the optimum solution for power minimization is given as in (18).

$$
\min g\left(\mu_{1}, \mu_{2}\right)=\sum\left(K_{1 i}+K_{2 i}\right)
$$

The best significance of maximization aimed at sets of $\mu_{1}$ and $\mu_{2}$ which describes the optimal operation $g\left(\mu_{1}, \mu_{2}\right)$ and $\beta$ is the Rytov variance, then the complexity is solved through the subsequent iterative process:

$$
\begin{aligned}
& \mu_{1, i, j}(t+1)=\mu_{1, i, j}(t)-\beta\left(t_{j, i}(t)-t_{i}(t)^{*}\right) \\
& \mu_{1, i, j}(t+1)=\mu_{1, i, j}(t)-\beta\left(p_{j, i}(t)-p_{i}(t)^{*}\right)
\end{aligned}
$$

As a final point, greedy algorithm is used for allocating power. After initializing the parameter values, all base stations resolves the problem of local maximization, then directs its response towards the associated BSs. All base stations enhance the event prices $\mu$ iteratively, and then direct another value to the BS. The iteration is continued until it reaches the termination condition.

\section{Atmospheric turbulence mitigation techniques:}

Optical scintillations [30] could be minimized through improving the collection area of the receiver side and it produces an integration of numerous strength of incident light on certain section of the receiver area. This strategy is represented as aperture averaging. Using this result into the scintillation index aimed at the spherical wave is with reference to the Rytov variance. 


$$
\sigma_{n}{ }^{2}=\exp \left[\frac{0.49 \beta^{2}}{\left(1+0.18 D^{2}+0.56 \beta^{12 / 5}\right)^{7 / 8}}+\frac{0.51 \beta^{2}\left(1+0.69 \beta^{12 / 5}\right)^{-12 / 5}}{1+0.90 D^{2}+0.62 D^{2} \beta^{12 / 5}}\right]-1
$$

Where $\beta$ is Rytov variance for the signal is, $D$ is the wave number and $\mathrm{Cn}^{2}$ is the refractive-index structure parameter. Near-ground horizontal-path propagation is assigned to the homogeneous turbulent field, then it has the constant refractive-index structure parameter. During weak fluctuations, the intensity is log normally dispersed. The lognormal distribution is often proposed while it uses an aperture averaging. The PDF of a lognormal variable $p$ with small variance is characterised as in the following equation.

$$
f(p)=\frac{1}{I \sqrt{2 \pi \sigma_{n}^{2}}} \exp \left\{\frac{\left[\ln \left(p_{i} /\left\langle p_{i}\right\rangle\right)+\sigma_{n}^{2} / 2\right]^{2}}{2 \sigma_{n}^{2}}\right\}
$$

Power loss is minimized for the scintillation and also it is the redistribution of intensity. Here broadening of the beam due to the turbulence is not considered. In addition to this we have modelled one filter namely modified Kalman filter.

Modified Kalman Filter for reducing scintillation noise.

A Modified Kalman filter is used for minimizing the scintillation noise due to the atmospheric turbulence and it is more compatible with the noise reduction. A State equation for the Kalman filter is given as in equation (23).

$$
v_{n}=A v_{n-1}+r_{n-1}
$$

From the above expression, we can deliberate the linear state vector which is given as $v_{n}, A$ is a state transfer matrix and $r_{n}$ denotes the white Gaussian noise with the zero mean random process.

Here intervals among two symbols are used for sampling the signals and it is represented as $\Delta t$. Then the expression of linear vector state is given as in (24).

$$
v_{n}=\left[\mu_{n} \sigma_{n}^{2} \frac{\partial \mu_{n}}{\partial t} \cdot \Delta t \frac{\partial \sigma_{n}^{2}}{\partial t} \cdot \Delta t\right]^{T}
$$

Here variance and mean of the sampled signal are denoted as $\sigma_{n}$ and $\mu_{n}$. Then the equation for the filtering measurement is given as in (25).

$$
y_{n}=I v_{n}+\mu_{n}
$$

According to the filtering measurements, $\mu_{n}$ is the noise power vector and I is the identity matrix, then the filtering measurement is also estimated with the estimated mean and standard deviation which are denoted by $\hat{\mu}_{n}, \hat{\sigma}_{n}$.

$$
y_{n}=\left[\begin{array}{llll}
\hat{\mu}_{n} & \hat{\sigma}_{n}^{2} & \hat{\mu}_{n}-\hat{\mu}_{n-1} & \hat{\sigma}_{n}^{2}-\hat{\sigma}_{n-1}^{2}
\end{array}\right]^{T}
$$

Where $\hat{\mu}$ is the mean of the sampled signal and $\hat{\sigma}_{n}^{2}$ is the variance of the sampled signal. An identity matrix of size $4 \times 4$ is generated for error removal and then gain of the filter is estimated in order to generate a priori. In this measurement process $\mu_{\mathrm{n}}$ is an AWGN vector, and next to this gain related to the Kalman filter is given below:

$$
K A_{n}=P_{i}^{-} I^{T}\left(I P_{i}^{-} I^{T}+N_{n}\right)^{-1}
$$

The sequential Kalman filter accurately stabilises the changing circumstance since the Kalman gain does not need a steady constant. Kalman gain, $P_{i}^{-}$is the power of the state error and it is achieved through solving the Riccati matrix equation, $N_{n}$ is the filtered state-error correlation matrix. In this above equation error covariance matrix is given as in eqn. (28)

$$
P_{n}^{-}=A P_{n-1} A^{T}+q_{n_{1}}
$$

As per the eqn. (26) error covariance is the function of process random vector and measurement noise vector. Then the estimated state equation is the function of gain in Kalman filter. Where the measurement noise variance $N_{n}$ and process noise variance $q_{n}$. Small but non-zero variances has maximum flexibility in 'tuning' the filter, as in [34].

$$
\hat{y}_{n}=y_{n}^{-}+N_{n}\left(v_{n}-I y_{n}^{-}\right)
$$

Then the priori estimation of the signal variance can be modelled with the exponential function (30).

$$
\hat{\sigma}_{n}^{2}=\exp \left(4 \hat{\sigma}_{x}^{2}\right)-1
$$

The estimated variance of signal $\hat{\sigma}_{n}^{2}$ is possible to calculate with (31), the variance of log-amplitude fluctuation is $\hat{\sigma}_{y}^{2}$, thus an optimal decision threshold fluctuates through the change of $\hat{\sigma}_{y}^{2}$, and then adaptive decision threshold is required for FSO links via atmospheric turbulence and then variance estimation of fluctuation in log-amplitude is written as in (31).

$$
\text { Where } \hat{\sigma}_{x}^{2}=0.25 \ln \left(\hat{\sigma}_{y}^{2}+1\right)
$$

It is possible to obtain the estimate of variance in optimum and threshold with optimum solution is set in FSO communication which efficiently reduces the atmospheric turbulence. 


$$
S N R=\frac{\hat{\mu}_{n}^{2}}{\hat{\sigma}_{n}^{2}}
$$

The SNR for computing Kalman filter's adaptive decision threshold is given in equation (32). SNR is the ratio of total estimated signal power to the estimated noise variance and this is obtained by computing the noise presented at the propagation. Then the variance of total noise is the sum of all the noises presented at the path. Finally SNR ratio should be minimized to show the elimination of atmospheric turbulence in FSO.

The degradation in the quality of the received signal is caused by the atmospheric channel that deteriorate the BER performance of the FSO system. Numerous types of mitigation techniques are employed to improve the reliability of the FSO system for all weather conditions [13]. The choice of modulation schemes depends on two main criteria: optical power efficiency and bandwidth efficiency in FSO communication. By computing optical power gain over OOK provided both the modulation schemes have a same Euclidean distance $d_{\min }$, the optical power efficiency can be measured. Power efficient modulation schemes are simpler to implement and are quite effective in mitigating the effect of the turbulence for low data rates. Maximum data for a given link length with a particular modulation scheme is determined by means of bandwidth efficiency. In general, the FSO communication supports a variety of binary and multilevel modulation formats. Among these two formats binary level format is most commonly utilized due to its simplicity and high power efficiency. The OOK modulation scheme requires an adaptive threshold in turbulent atmospheric conditions for best outcomes [36]. Algorithm for CROF methodology is given in table 2 .

Table 2. Algorithm for CROF methodology

\begin{tabular}{|ll|}
\hline 1 & Initialize source, relay and destination set \\
2 & Estimate distance between source and destination \\
3 & Select relay according to switching threshold \\
4 & Set maximization problem Karush-Kuhn-Tucker $(\mathrm{KKT})$ \\
5 & Set $\mathrm{t}=0$, reset $\mu_{1, i, j}(0), \mu_{2, i, j}(0) \gamma 1, \mathrm{i}, \mathrm{j}(0), \gamma 2, \mathrm{i}, \mathrm{j}(0)$ to \\
& some value \\
6 & while end condition not encountered \\
7 & End if \\
8 & Every BS resolves maximization problem locally then \\
& transmits result to associated BSs \\
9 & Update $t \leftarrow t+1$ \\
10 & Estimate state equation in Kalman filtering \\
11 & Estimate filtering measurement \\
12 & Calculate SNR for reporting noise percentage \\
13 & End \\
\hline
\end{tabular}

For an optimum function in atmospheric conditions, it needs a numerous FSO system with high efficiency. In addition to that it should overcome an attenuation fluctuation problem and for that it needs a large power link margin. Thus an essential power link margin could in various cases be certified through short link distances. The fade affected through scintillations looks only if the link distance is moderately enormous, and these schemes are not affected by turbulence.

\section{EXPERIMENTAL RESULTS AND DISCUSSIONS}

In this section, minimum atmospheric turbulence is achieved with minimum scintillation noise. If we minimize the scintillation noise then we can reduce the effect of turbulence. Because atmospheric turbulence induced scintillation noise presented at the signal. Scattering is also one of the FSO challenge and in this work we have concentrated on scattering minimization through minimum BER. Here power loss is also taken into account with suitable relay selection algorithm. In our work, we consider the effect of relay selection algorithm, turbulence minimization, attenuation minimization. Here the relay selection is based on the combination of the AF/FSO system with optimal relaying. We explain the mathematical outcomes for outage evaluation, which is attained over the resulting terms and MATLAB simulations. For that, set the FSO system with signal wavelength as $1.55 \mu \mathrm{m}$ and attenuation coefficient as 0.43 $\mathrm{dB} / \mathrm{Km}$. The total transmission length required for the transmission is $0.5 \mathrm{Km}$ and maximum transmitter power of the nodes is $0.5 \mathrm{~W}$. Audio signal's bandwidth is $10 \mathrm{MHz}$ and data rate is $3 \mathrm{Gbps}$ in which FSO system has the space loss of $1.09 \times 10^{-26}$. Atmospheric turbulence margin is set as $-11.30 \mathrm{~dB}$ and the gain of transmitter antenna is $3.73 \times 10^{8}$. Here transmitter optical loss is 0.1 factors, clear air transmission loss is -2.08 and link margin is $-6 \mathrm{~dB}$. For serial relaying, we assign the successive nodes are identical distance beside the path from the source to the destination. The total number of relay for the transmission is $\mathrm{N}=1,2,3 \ldots \mathrm{n}$.

This experiment is expected to reduce the environmental challenges with the proposed algorithm. Table 3 given the Parameters and condition for the experiment.

Table 3. Parameters and condition for the experiment

\begin{tabular}{|c|c|}
\hline Parameters & value \\
\hline Signal wavelength & $1.55 \mu \mathrm{m}$ \\
\hline attenuation coefficient & $0.43 \mathrm{~dB} / \mathrm{Km}$ \\
\hline Total transmission length & $0.5 \mathrm{Km}$ \\
\hline maximum transmitter power of the nodes & $0.5 \mathrm{~W}$ \\
\hline Audio signal's bandwidth & $10 \mathrm{MHz}$ \\
\hline data rate & $3 \mathrm{Gbps}$ \\
\hline space loss & $1.09 \times 10^{-26}$ \\
\hline Atmospheric turbulence margin & $-11.30 \mathrm{~dB}$ \\
\hline gain of transmitter antenna & $3.73 \times 10^{8}$ \\
\hline transmitter optical loss & 0.1 \\
\hline clear air transmission loss & -2.08 \\
\hline link margin & -6 \\
\hline Environment conditions & Fog, haze, rainy, \\
\hline
\end{tabular}

\section{A. Quality measures}

Some of the quality measures are evaluated to show the performance of the proposed free space optical communication.

\section{Signal to noise ratio $(S N R)$}

Signal to Noise Ratio is the ratio of total signal power 
to the total noise power and this total noise power is obtained by computing the noise presented at the propagation. Then the variance of total noise is the sum of all the noises presented at the path as (32).

\section{Bit Error Ratio (BER)}

Bit Error Ratio is the incorrect signal bit which is incorrect during the transmission and in order to generate the error ratio of this signal bit is divided by the total number of bits in the signal. The expression of BER for Intensity Modulation Direct Detection (IM/DD) can be expressed as

$$
B E R=0.5 \operatorname{erfc}\left[\frac{\sqrt{S N R}}{2 \sqrt{2}}\right]
$$

\section{Throughput}

It measures the rate of signal that entirely transmitted over the communication path. It is also defined as the signals send to the time taken for transmitting the corresponding signals from the receiver.

$$
\text { Throughput }=\frac{\text { signal sending rate }}{\text { time taken to transmit the signal }}
$$

\section{Root Mean Square Error (RMSE)}

Root Mean Square Error is the prediction error and it is calculated from the standard deviation of the signal.

$$
R M S E=\sqrt{1-c^{2} \sigma_{n}}
$$

Where, $\sigma_{\mathrm{n}}$ is the standard deviation of the received signal and ' $\mathrm{C}$ ' is the correlation coefficient.

\section{Optical power loss}

Optical power is measured from the source and destination power level and it is the difference between source powers to the destination power. It is measured in terms of $\mathrm{dBm}$.

$$
\text { optical power loss }=\text { source power }- \text { destination power }
$$

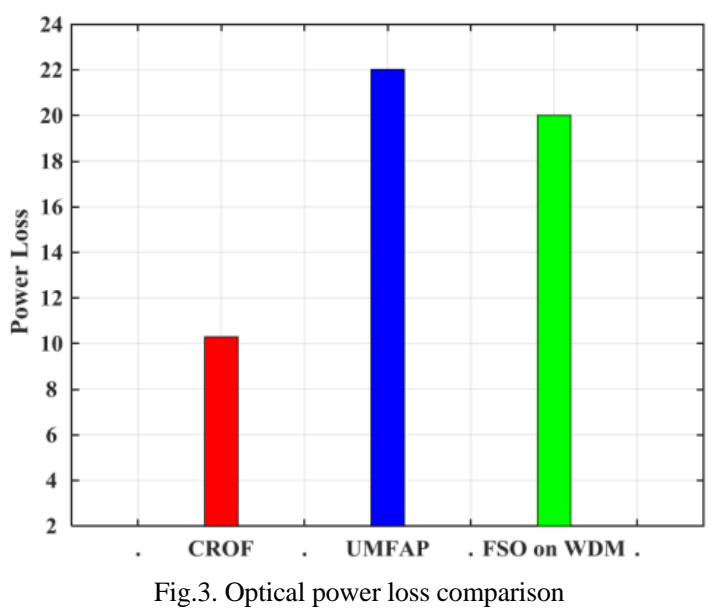

Optical power loss (figure 3) is measured in between source and destination. It is dependent upon the source and the destination power. For a better system, optical power loss should be low and in our case proposed power loss of $10.29 \mathrm{~dB}$ and existing work has some more power loss of $22.014 \mathrm{~dB}$. Thus, our proposed work is better in case of power consumption.

\section{B. Evaluation of quality factors under different environmental conditions}

Here, we evaluated the system in the atmospheric conditions like haze, rainy, fog and clear weather condition [14] and the data set is taken from https://www.kaggle.com/mahirkukreja/delhi-weatherdata\#testset.csv.

\section{Signal evaluation under Haze}

Throughout hazy days, the attentiveness of particulate matter impurities is lesser than 10 microns. Source apportionment through substance mass stability receptor modeling mentions that air pollutants could pass a long distance and expressively change the air of the downwind area. Therefore, every day through PM10 beyond 100 $\mu \mathrm{g} / \mathrm{m} 3$ are recognized as hazy days. Visibility was condensed through an average of $7.0 \mathrm{~km}$ throughout haze days through the lowest visibility verified $(0.5 \mathrm{~km})$ on the same day by means of the maximum PM10 attentiveness; Such data are statistically evaluated for determining the real scattering effectiveness of the maximum secondary aerosol species. The separate scattering competences are then castoff for estimating the contributions of main source of volatile gases for reducing visibility [31].

Due to the forest burning haze weather condition, the recorded visibility range of the FSO system is 200m [17]. Compared to other environmental conditions haze stay longer time in the air. Thus, throughout the designing process, attenuation of haze must be taken into account for the link design. So, in hazy days all the atmospheric attenuations are wavelength (upto 500m there is no effects) [32] dependent. This attenuation may introduce some losses in the system and for our proposed work, the attenuation should be low. Here the proposed work (CROF) is compared with the Unified empirical Model for Fog Attenuation Prediction (UMFAP) [25] and FSO on WDM [27].

Figure 4 evaluates the performance measures under haze condition. Except to the throughput (d) other three evaluations like BER (a), RMSE (b) and SNR (c) achieves minimum values than the existing work which shows better performance of the proposed work. Throughput has maximum performance under different atmospheric attenuation. Fig. 4(a) shows the ergodic link for several haze conditions. Again, the performance of FSO system under RMSE (fig. 4(b)) is better regardless the wavelength and the transmitted power. The same performance can be noticed for SNR (figure 4 (c)). However, for longer link lengths, the capacity enhances quite well. For example, for L $0.2,0.3,0.4$ and $0.5 \mathrm{~km}, \mathrm{SNR}$ is elaborately reduced. For throughput under haze condition (fig. 4 (d)) is much better than other environmental conditions. If we fix the 
transmitted power to $22 \mathrm{dBm}$ and attenuation of 10 , then we can achieve $13 \mathrm{MBps}$ (CROF) and which is high while comparing to the existing two works. The SNR and data rate is evaluated in terms of link range and it is calculated for $0.5 \mathrm{~km}$ range. In addition to this throughput is also measured in terms of atmospheric attenuation coefficient $(\mathrm{dB} / \mathrm{Km})$.

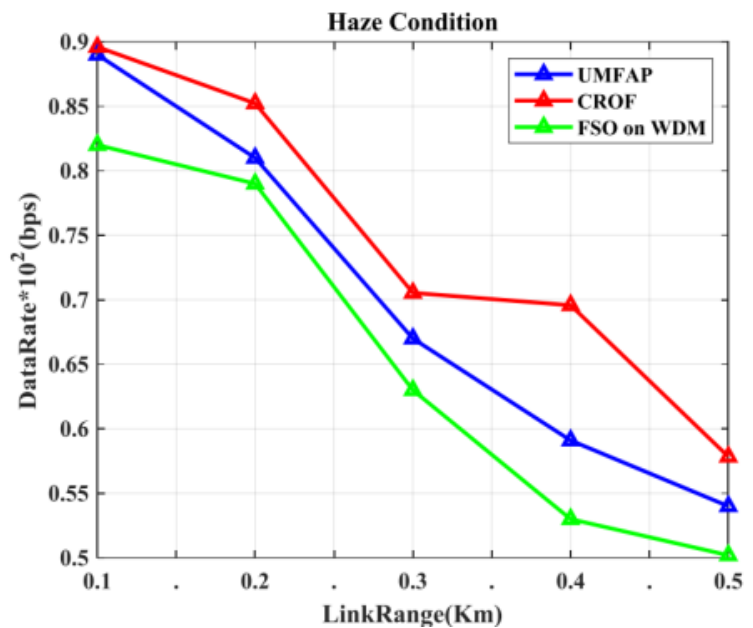

Fig.4(a). Data rate vs Link range

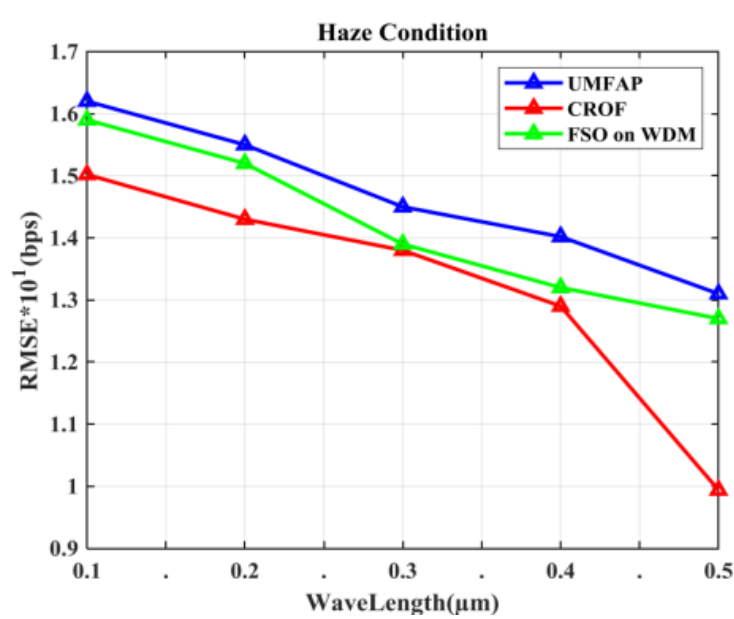

Fig.4(b). RMSE vs Wave length

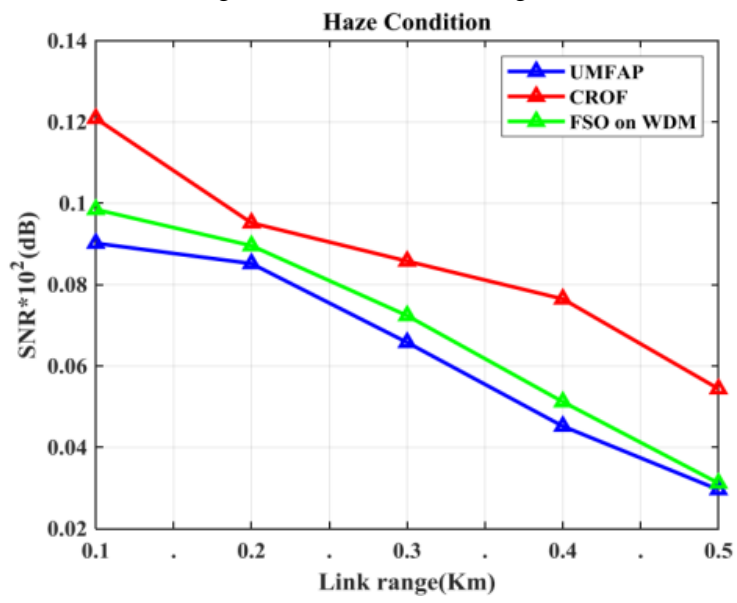

Fig.4(c). SNR vs Link range

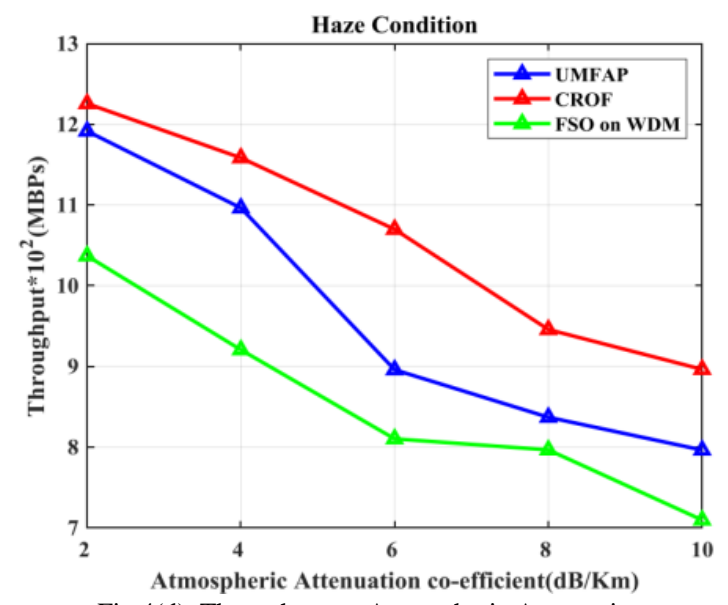

Fig.4(d). Throughput vs Atmospheric Attenuation

Fig.4. Different factors under Haze condition

\section{Signal evaluation under Fog}

In case of fog condition, the visibility comes down and humidity of the system has the range of hundred percentage saturation level. Therefore, contribution of absorption and the whole attenuation coefficient is small while associating to the effect of scattering.

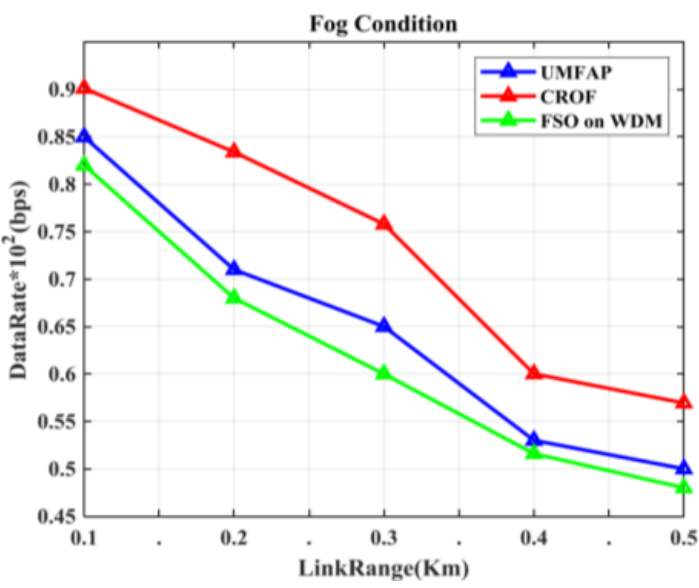

Fig.5(a). Data rate vs Link range

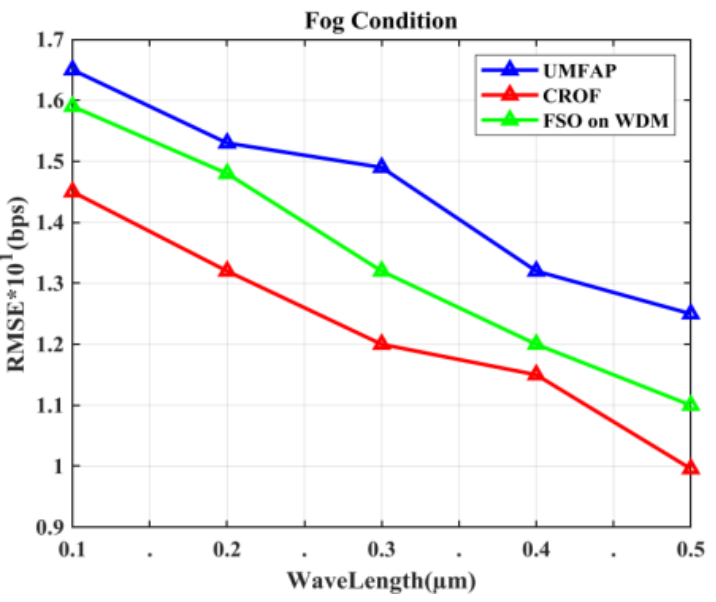

Fig.5(b). RMSE vs Wave length 


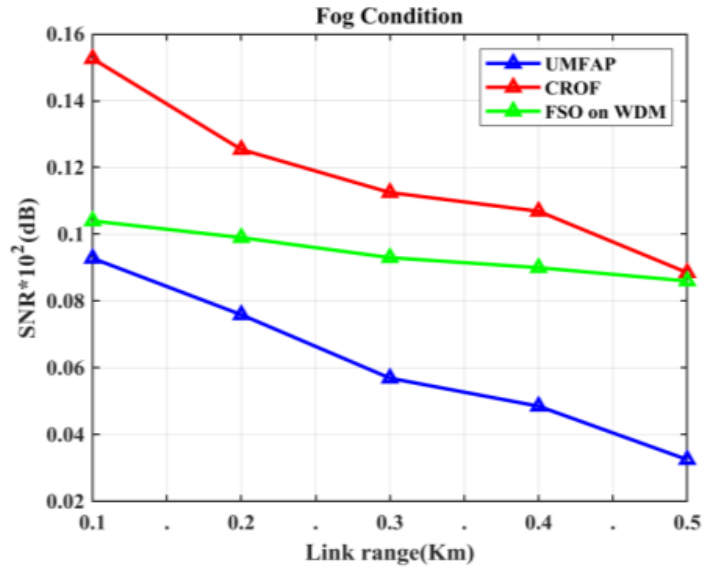

Fig.5(c). SNR vs Link range

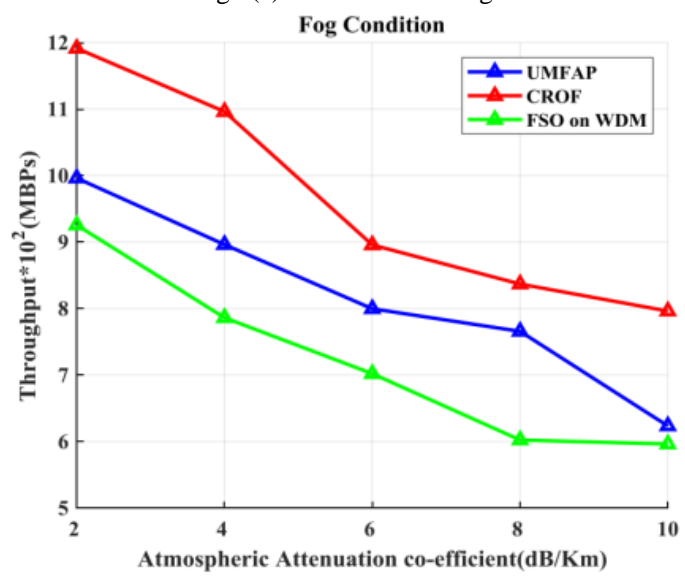

Fig.5(d). Throughput vs Atmospheric Attenuation

Fig.5. Different factors under Fog condition

So, in optical communication scattering effect will predict the attenuation which is done with minimum BER. Figure 5 (a) and 5 (c) represented the BER and SNR, which is optimizely reduced for the different link range. RMSE (b) also reduced for the number of wavelength under fog condition. Fig. 5(a) shows the ergodic link for several foggy conditions. Again, the performance of FSO system under RMSE (fig. 5(b)) is good regardless the wavelength and the transmitted power. For example, for the attenuation of 2, 4, 6, 8 and10, throughput achieves $25 \%$ better performance than the existing works. For throughput under fog condition (fig. 5 (d)) is much better. If we fix the attenuation coefficient to 10 and, then we can achieve $12 \mathrm{Mbps}$ (CROF) which is high while compared to the existing two works.

\section{Signal evaluation under rainy}

Under the condition of monsoon, the parameter of weather variations from $1 \mathrm{~ms}^{-1}$ to $7.2 \mathrm{~ms}^{-1}$ through the standard of $1.59 \mathrm{~ms}^{-1}$ for wind speed, $27-39{ }^{\circ} \mathrm{C}$ through $3.75^{\circ} \mathrm{C}$ for temperature, $25-84 \%$ with the std of $15.89 \%$ for qualified humidity and $2-10 \mathrm{~km}$ with the std of 2.19 $\mathrm{km}$ for visibility. An alternative situation of the atmosphere detected on 6 June 2013 (Thursday) which is scattered cloudy, partial cloudy and mostly cloudy, light drizzle, and light rainy and hazy. The experimental time series plot of attenuation predicted from local meteorological and visibility data collected on 6 June
2013 and measured values. The max-min values of measured attenuation are $\approx 6.45 \mathrm{~dB} / \mathrm{km}$ and $1.74 \mathrm{~dB} / \mathrm{km}$ with the std of $1.29 \mathrm{~dB} / \mathrm{km}$. A short interval of high attenuation $\approx 6.45 \mathrm{~dB} / \mathrm{km}$ is seen in the time span of 3.00 4.00 p.m due to the normal wind speed and high temperature and low relative humidity. The low and high attenuation of $\approx 1.68 \mathrm{~dB} / \mathrm{km}$ and $\approx 8.40 \mathrm{~dB} / \mathrm{km}$ with the std of $\approx 1.19 \mathrm{~dB} / \mathrm{km}$ when the visibility is high [33]. Attenuation is also happening when the optical signal is transmitted through the rainy season [27]. The major factor of attenuation in optical link is rain droplets and it may enforce attenuations for certain links.

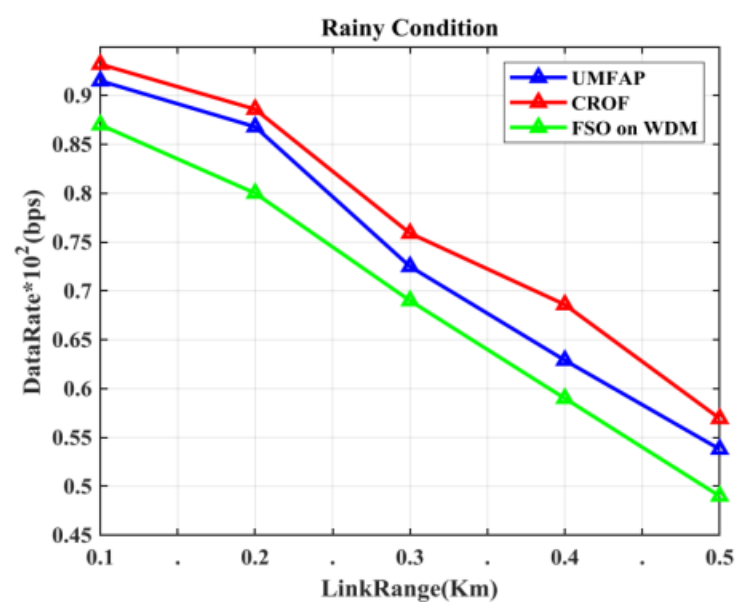

Fig.6(a). Data rate vs Link range

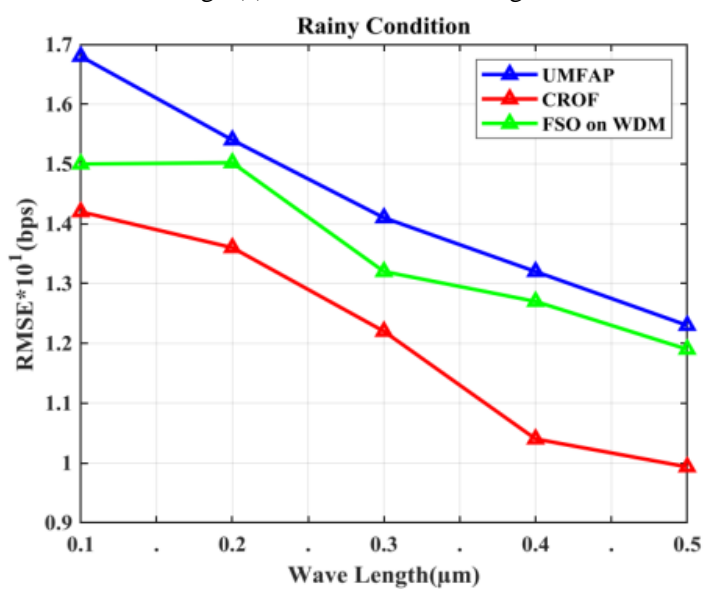

Fig.6(b). RMSE vs Wave length

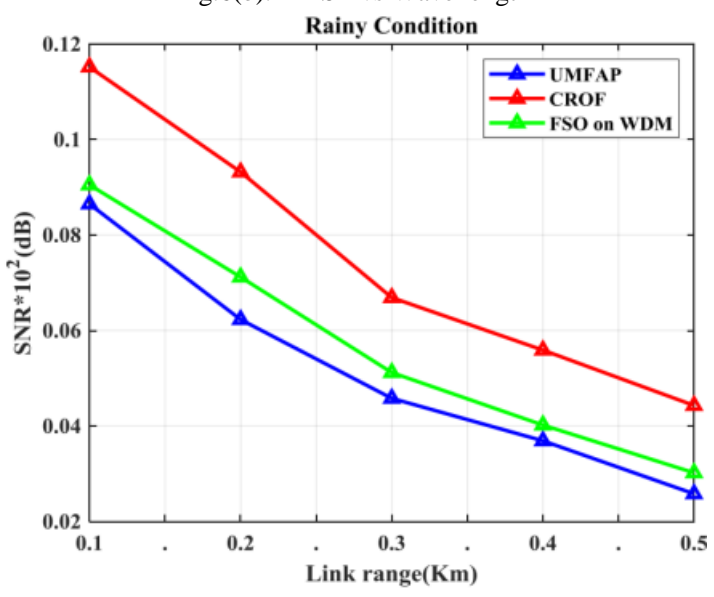

Fig.6(c). SNR vs Link range 


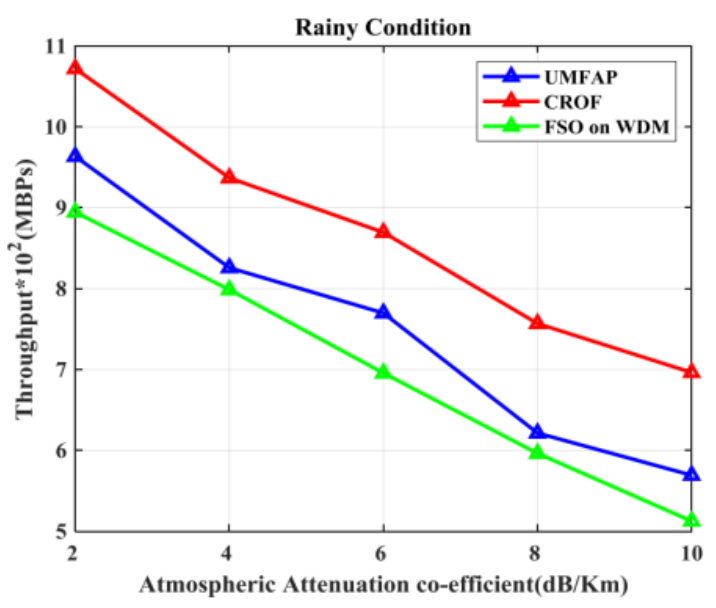

Fig.6(d). Throughput vs Atmospheric Attenuation

Fig.6. Different factors under Rainy condition

In figure 6 (a), (b), (c) and (d) has the enhanced performance under rainy condition. In some cases, refraction and reflection may induce due to the size of rain droplets. If it is large means it produces some irregularities in the FSO link by scattering. So our proposed work attains maximum results while compared to the existing work. Fig. 6(a) shows the ergodic link for several rainy conditions. Again, the performance of FSO system under RMSE (fig. 6(b)) is good regardless the wavelength and the transmitted power. For example, for the attenuation of 2, 4, 6, 8 and10, throughput achieves $30 \%$ better performance than the existing works. For throughput under rain condition (fig. 6 (d)) is much better. If we fix the attenuation coefficient of 10 and, then we can achieve $10.82 \mathrm{MBps}$ (CROF) and which is high while compared to the existing two works.

\section{Signal evaluation under clear weather condition}

In clear weather condition, visibility range is $5 \mathrm{~km}$ and the wavelength for the signal propagation is $650 \mathrm{~nm}$. In this clear weather condition turbulence effect is introduced and this will produce changes in the atmospheric refractive index. Since scintillation and random phase change is introduced at the receiver. In addition to the scintillation, some pointing error is occurring at the transmission link.

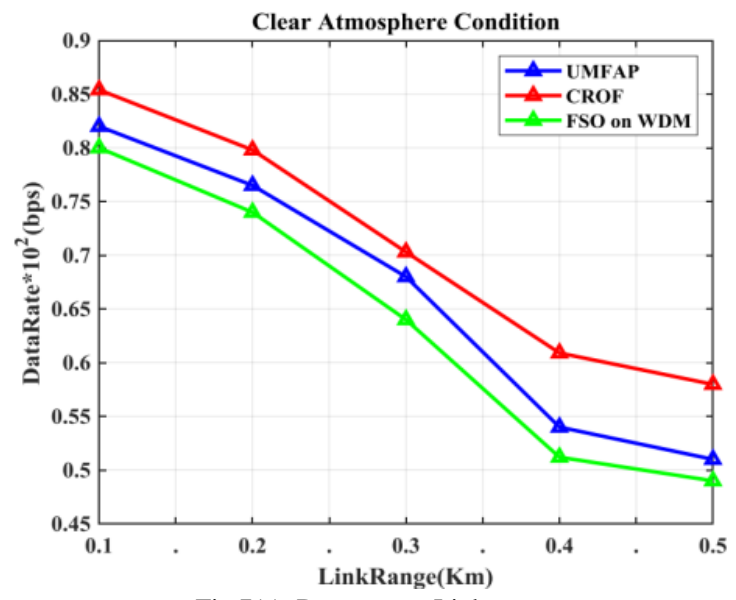

Fig.7(a). Data rate vs Link range

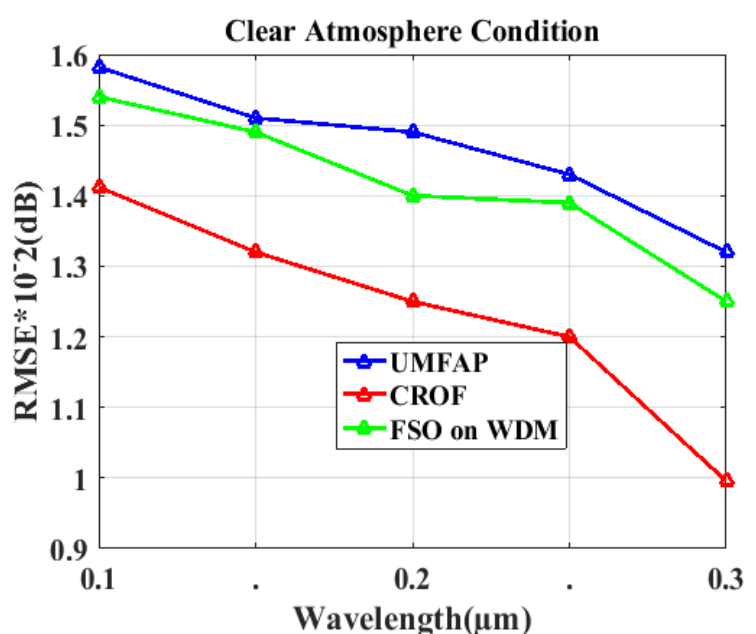

Fig.7(b). RMSE vs Wave length

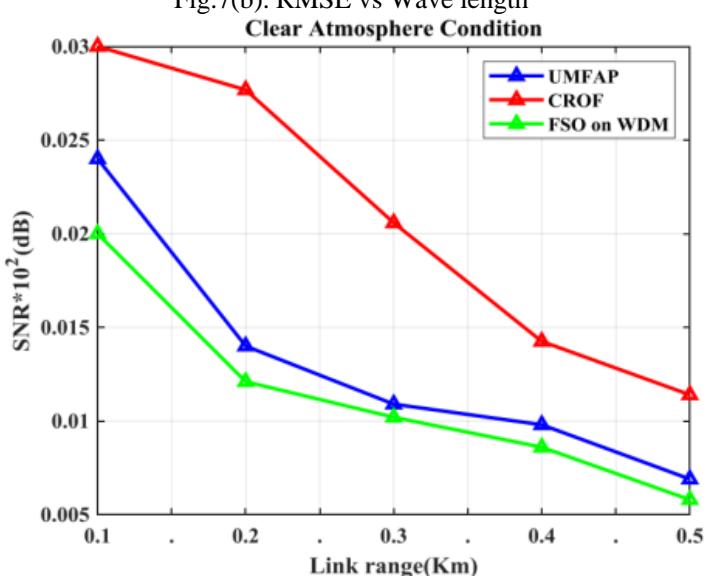

Fig.7(c). SNR vs Link range

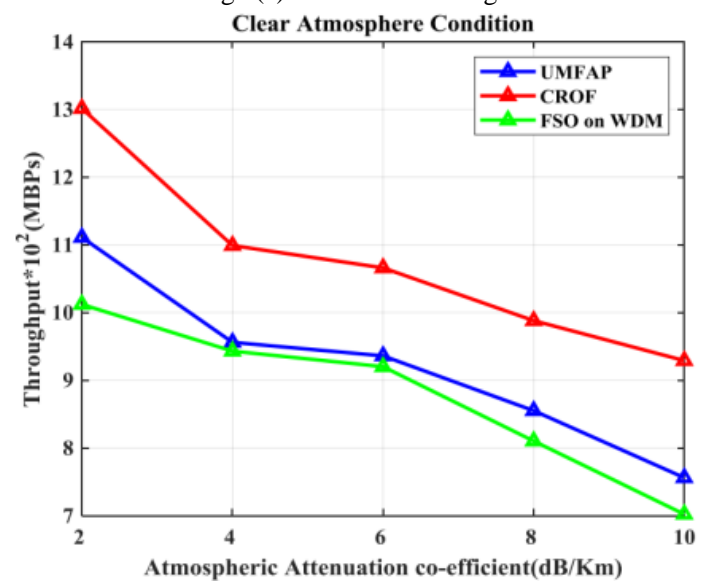

Fig.7(d). Throughput vs Atmospheric Attenuation

Fig.7. Different factors under clear weather condition

From figure 7 (a), (b), (c) and (d), the result obtain for the proposed work is better in case of BER, SNR, RMSE and throughput. In case of throughput, in reference to the atmospheric attenuation coefficient, throughput is increased over the clear weather condition. Amongst entire occurrences, fog gives the maximum consequences thus it is established of little water droplets have the measurements closer to the span of infrared wavelengths. Fig. 7(a) shows the ergodic link for several atmospheric conditions. Again, the performance of FSO system under RMSE (fig. 7(b)) is good regardless the wavelength and the transmitted power. For throughput under clear weather 
condition (fig. 7 (d)) is much better. If we fix the attenuation coefficient of 10 and, then we can achieve 13 MBps (CROF) and which is high while compared to the existing two works.

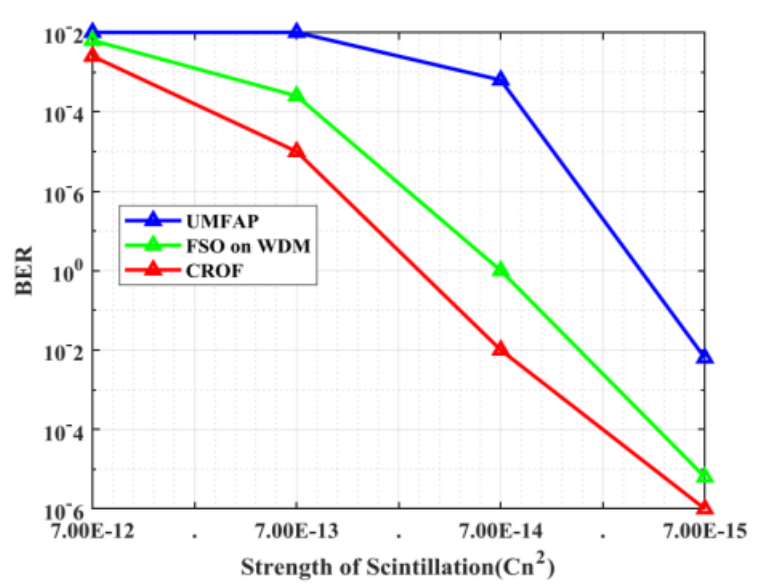

Fig.8. Scintillation vs BER measurement

The figure 8 , represented that $\mathrm{CROF}$ technique functions better than the FSO on WDM Detection techniques for minimum ranges of $\mathrm{Cn}^{2}$ i.e. $7 \mathrm{e}^{-14}$ and $7 \mathrm{e}^{-15}$. In case of UMFAP, modest value of $\mathrm{Cn}^{2}$ i.e. $7 \mathrm{e}^{-13}$ the CROF till represented suitable outcomes through minimizing the BER from $3.33 \mathrm{e}^{-1}$ to $1.55 \mathrm{e}-5$. In case for maximum value of $\mathrm{Cn}^{2}$ i.e. 7e-12, even the FSO on WDM methods cannot help more because the BER varies minimally from $4.45 \mathrm{e}^{-1}$ to $1.42 \mathrm{e}-1$. In which atmospheric structure constant $\mathrm{Cn}^{2}$ is a quality of the strength of the scintillation, $\mathrm{k}=2 \pi / \lambda$, and $\mathrm{L}$ is the link length. The atmospheric structure constant characteristically proceeds values from 10-12 and higher. Depends on the climatic conditions the parameters will vary. As the summary, the results of the proposed work is achieved the above mentioned objectives (i.e) we can achieved an efficient FSO system with minimum atmospheric turbulence and this minimum atmospheric turbulence is achieved with minimum scintillation. From the figure 8 , we can show the BER vs Scintillation performance is better than other techniques since it yields minimum value than other techniques. Also in optical communication scattering effect is predict the attenuation which is done with minimum BER. In figure 4 to 7 , all the BER performance under different environment condition represented that we achieved minimum BER value as the result the scattering effects also reduced. In addition to that, an efficient relay selection should reduce the power loss. Figure 3, shows the minimum power loss than other techniques since our proposed relay section scheme is an efficient one.

\section{CONCLUSION}

In this paper, we have considered the FSO throughput and high data rate under the constriction of limited BER and SNR. This section deliberates specified hypothetical evaluation of common factors for originating optical communication that has to function under numerous atmospheric conditions, such as haze, rainy, foggy and clear weather conditions. In this paper, we considered the challenging problem of relay selection and proper atmospheric turbulence management. We developed effective method for minimizing scattering losses and this minimized the atmospheric turbulence. Also we maximized the total throughput of the cooperative FSO network and one of the major advantage is the scintillation noise removal. So compared to other techniques we have using modified Kalman filter based scintillation noise removal techniques since we achieved minimum Bit Error Rate (BER) than UMFAP and FSO on WDM.

Here we use a limited amount of transceivers with limited power source for the free space transmission. Our model shows that the proposed centralized algorithm attained the highest ability for the reliable FSO transmission. The centralized relay selection algorithm needs a central controller for getting more amounts of data at the receiver. The proposed distributed algorithm can be implemented towards accomplishing a greater enactment over the existing scheme in case of minimum error ratio. For that modelled, the numerical models for numerous link ranges under several environments are specified to demonstrate the developments in environmental factors influence performance and to validate system model optimization. Final performances are evaluated in terms of power loss, signal to noise ratio, throughput, bit error rate and root mean square error. These factors are evaluated under various environmental conditions to show the CROF efficiency.

\section{Future work:}

Advantages of FSO communication with its application have some challenges emerging because of the medium weakening. FSO system signifies few issues termed as decreasing in medium which may affect the execution of communication as power loss. Many researches are going in order to overcome an impact in the system configuration. Numerous models depend on these practices which will utilize to consider the execution of system before presenting it at the area. This will prompt the system improvement.

\section{REFERENCES}

[1] D. Keddar and S. Arnon, "Urban optical wireless communication networks: the main challenges and possible solutions," IEEE Opt. Commun., vol. 42, no. 5, pp. 51-57, May 2004.

[2] A. K. Majumdar, "Free-space laser communication performance in the atmospheric channel," L. Opt. Fiber Commun. Rep., vol. 2, pp. 345-396, 2005.

[3] L. Andrews, R. L. Philips, and C. Y. Hopen, Laser Beam Scintillation with Applications. SPIE Press, 2001.

[4] M. A. Al-Habash, L. C. Andrews, and R. L. Phillips, "Mathematical model for the irradiance pdf of a laser beam propagating through turbulent media," Opt. Eng, vol. 40, no. 8, pp. 1554-1562, 2001.

[5] Ciaramella E, Arimoto Y, Contestabile G, Presi M, D'Errico A, Guarino V, and Matsumoto M. 1.28 Terabit/s (32x40 Gbit/s) WDM transmission system for free space 
optical communications, IEEE Journal on selected areas in communications, 2009;27(9).

[6] E. Bayaki, Schober, R and. Mallik R K. Performance analysis of MIMO free-space optical systems in gammagamma fading, IEEE Transactions on Communications, 2009; 57(11): 3415-3424.

[7] Thakur, Aditi, S. Nagpal, and A. Gupta. Kerr effect based spectrum sliced wavelength division multiplexing for free space optical communication. Optik-International Journal for Light and Electron Optics, Elsevier, 157 (2018) 31-37.

[8] Kaushal, Hemani, V.K. Jain, and S. Kar. Free Space Optical Communication. Springer, 2017.

[9] Ninos M P, Nistazakis HE, and Tombras G S. On the BER performance of FSO links with multiple receivers and spatial jitter over gamma-gamma or exponential turbulence channels. Optik-International Journal for Light and Electron Optics, Elsevier, 2017; 138: 269-279.

[10] Vu, Minh Q, Nguyen NTT, Pham HTT. And Dang NT. All-optical two-way relaying free-space optical communications for HAP-based broadband backhaul networks. Optics Communications, Elsevier, 2018; 410: 277-286.

[11] Prabu K, Bose S. and Kumar DS. BPSK based subcarrier intensity modulated free space optical system in combined strong atmospheric turbulence, Optics Communications, 2013; 305(1): 185-189.

[12] Sandalidis HG, Tsiftsis TA, Karagiannidis GK. and Uysal M. BER performance of FSO links over strong atmospheric turbulence channels with pointing errors, IEEE Communications Letters, 2008; 12(1): 44-46.

[13] Kaushal, Hemani and Kaddoum G. Optical communication in space: Challenges and mitigation techniques. IEEE Communications Surveys \& Tutorials 2017; 19(1): 57-96.

[14] Mansour, Ali, Mesleh R. and Abaza M. New challenges in wireless and free space optical communications. Optics and Lasers in Engineering, Elsevier, 2017; 89: 95-108.

[15] Sharma, Manish, Chadha D. and Chandra V. Performance enhancement of free space optical communication system using beam-optimized serial relaying in saturated atmosphere with pointing errors. Photonic Network Communications2017; 33(2): 208-216.

[16] Alheadary, Park WK-H. And M-Slim Alouini. Performance analysis of multihop heterodyne free-space optical communication over general Malaga turbulence channels with pointing error. Optik-International Journal for Light and Electron Optics 2017; 151: 34-47.

[17] Sharma, Manish, Chadha D. and Chandra V, Performance enhancement of free space optical communication system using beam-optimized serial relaying in saturated atmosphere with pointing errors. Photonic Network Communications, Springer, 2017; 33 (2): 208-216.

[18] Bayaki E, Michalopoulos DS. And R Schober, EDFAbased all-optical relaying in free-space optical systems, IEEE Transactions on Communications, 2012; 60(12): 3797-3807.

[19] Karimi M, and Nasiri-Kenari M. Free space optical communications via optical amplify-and-forward relaying, Journal of Lightwave Technology, 2011; 29(2): 242-248.

[20] Chen, Li and Wang W. Effective capacity of MIMO freespace optical systems over gamma-gamma turbulence channels. Optics Communications 2017; 382: 450-454.

[21] Ansari IS, Yilmaz and Alouini MS. Performance Analysis of Free-Space Optical Links over Málaga (\$\ mathcal $\{\mathrm{M}\}$ \$) Turbulence Channels with Pointing Errors, IEEE Transactions on Wireless Communications, 2016; 15(1): 91-102.
[22] Niaz A, Qamar F, Ali M, Farhan R, Islam MK. Performance analysis of chaotic FSO communication system under different weather conditions. Transactions on Emerging Telecommunications Technologies. 2019 Feb;30(2):e3486.

[23] Upadhya A, Dwivedi VK, Singh G. Relay-aided freespace optical communications using $\alpha-\mu$ distribution over atmospheric turbulence channels with misalignment errors. Optics Communications. 2018 Jun 1;416:117-24.

[24] Basahel A, Rafiqul IM, Habaebi MH. and Suriza AZ. Visibility effect on the availability of a terrestrial free space optics link under a tropical climate, Journal of Atmospheric and Solar-Terrestrial Physics, 2016;143(1): 47-52.

[25] Esmail MA, Fathallah H. and Alouini M-S. Outdoor FSO Communications under Fog Attenuation Modelling and Performance Evaluation, IEEE Photonics Journal, 2016; 8(4): 1-22.

[26] Sharma, Bansal A. and Garg P. Relay selection in mixed RF/FSO system over generalized channel fading, Transactions on Emerging Telecommunications Technologies, 33(2):143-151.

[27] Malik A. and Singh P. Comparative analysis of point to point FSO system under clear and haze weather conditions, Wireless personal communications, 2015;80(2): 483-492.

[28] Li Z. and Zhao X. Kalman Filter Based Optimal Controllers in Free Space Optics Communication, Journal of the Optical Society of Korea, 2016; 20(3): 368-380.

[29] Voelz D.G. and Xiao X. Metric for optimizing spatially partially coherent beams for propagation through turbulence. Optical Engineering, 2009; 48(3): 036001.

[30] Prokes A. and Brancik L. December. Degradation of free space optical communication performance caused by atmospheric turbulence. In Advances in Computational Tools for Engineering Applications (ACTEA), 2012 2nd International Conference on IEEE. 2012; 338-341

[31] Sahani M, Zainon NA, Mahiyuddin WRW, Latif, MT, Hod R, Khan, MF, Tahir, NM. And Chan CC. A casecrossover analysis of forest fire haze events and mortality in Malaysia. Atmospheric Environment, 2014; 96:257-265.

[32] Miglani, Rajan. Analysis of FSO communication links for mid and far infrared wavelengths. Int J Sci Eng Res 2013; 4.

[33] Raj AAB. And Selvi, J.A.V. Comparison of different models for ground-level atmospheric attenuation prediction with new models according to local weather data for FSO applications. Journal of Optical Communications, 2015; 36(2): 181-196.

[34] Chen C, Yang H, Jiang, H, Fan, J., Han, C. and Ding, Y. May. Mitigation of turbulence-induced scintillation noise in free-space optical communication links using Kalman filter. In Image and Signal Processing, 2008. CISP'08. Congress on IEEE.2008; 5: 470-473.

[35] M. A., Khalighi and M., Uysal, "Survey on Free Space Optical Communication: A Communication Theory Perspective", IEEE Communications Surveys \& Tutorials, 16(4), pp. 2231-2258, 2014.

[36] A. Viswanath, H. Kaushal, V. K. Jain, and S. Kar, "Evaluation of performance of ground to satellite free space optical link under turbulence conditions for different intensity modulation schemes," Proc. SPIE, Free Space Laser Comm. and Atmosph. Prop. (XXVI), vol. 8971, 2014

[37] Samimi H, Azmi P. Subcarrier intensity modulated freespace optical communications in K-distributed turbulence channels. Journal of Optical Communications and Networking. 2010 Aug 1;2(8):625-32 
[38] E. Jarangal and D. Dhawan, "Comparison of channel models based on Atmospheric turbulences of FSO systemA Review," pp. 282-286, 2018. IJRECE VOL. 6 ISSUE 1 JAN.-MAR. 2018 ISSN: 2393-9028 (PRINT) | ISSN: 2348-2281 (ONLINE)

\section{Authors' Profiles}

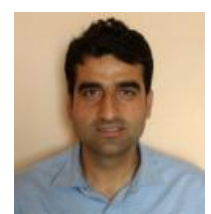

M Mubasher Hassan: Pursuing Ph.D from National Institute of Technology (NIT) Srinagar, J\&K, India. $\mathrm{He}$ is working as Assistant Professor at BGSB University Rajouri (J\&K)-India. He has received his M.Tech degree from the NIT Srinagar in 2007 and presently working as Assistant Professor at BGSB University Rajouri J\&K, India. He has received his B.E degree from the Jammu University in 2004.

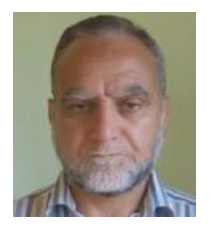

G M Rather has received his Ph.D degree from I.I.Sc Banglore. He is presently working as Professor in the department of ECE, National Institute of Technology (NIT) Srinagar J\&K, India. He has more than 35 years experience of teaching and research, particularly in the field of communication systems, computer networks and advanced wireless systems.

How to cite this paper: M. Mubasher Hassan, G M Rather, "Centralized Relay Selection and Optical Filtering Based System Design for Reliable Free Space Optical Communication over Atmospheric Turbulence", International Journal of Computer Network and Information Security(IJCNIS), Vol.12, No.1, pp.27-42, 2020. DOI: 10.5815/ijcnis.2020.01.04 